\title{
Adenosine augmentation ameliorates psychotic and cognitive endophenotypes of schizophrenia
}

\author{
Hai-Ying Shen, ${ }^{1}$ Philipp Singer, ${ }^{1}$ Nikki Lytle, ${ }^{1}$ Catherine J. Wei, ${ }^{2}$ Jing-Quan Lan, ${ }^{1}$ \\ Rebecca L. Williams-Karnesky, ${ }^{1,3}$ Jiang-Fan Chen, ${ }^{2}$ Benjamin K. Yee, ${ }^{1}$ and Detlev Boison ${ }^{1,3}$ \\ ${ }^{1}$ Robert Stone Dow Neurobiology Laboratories, Legacy Research Institute, Portland, Oregon, USA. 2Department of Neurology, Boston University \\ School of Medicine, Boston, Massachusetts, USA. ${ }^{3}$ Department of Neurology, Oregon Health and Science University, Portland, Oregon, USA.
}

\begin{abstract}
An emerging theory of schizophrenia postulates that hypofunction of adenosine signaling may contribute to its pathophysiology. This study was designed to test the "adenosine hypothesis" of schizophrenia and to evaluate focal adenosine-based strategies for therapy. We found that augmentation of adenosine by pharmacologic inhibition of adenosine kinase (ADK), the key enzyme of adenosine clearance, exerted antipsychotic-like activity in mice. Further, overexpression of ADK in transgenic mice was associated with attentional impairments linked to schizophrenia. We observed that the striatal adenosine $A_{2 A}$ receptor links adenosine tone and psychomotor response to amphetamine, an indicator of dopaminergic signaling. Finally, intrastriatal implants of engineered adenosine-releasing cells restored the locomotor response to amphetamine in mice overexpressing ADK, whereas the same grafts placed proximal to the hippocampus of transgenic mice reversed their working memory deficit. This functional double dissociation between striatal and hippocampal adenosine demonstrated in $A d k$ transgenic mice highlights the independent contributions of these two interconnected brain regions in the pathophysiology of schizophrenia and thus provides the rationale for developing local adenosine augmentation therapies for the treatment of schizophrenia.
\end{abstract}

\section{Introduction}

Despite extensive research over half a century, schizophrenia remains a major health concern, affecting more than one percent of the population. Two hypotheses, those of dopaminergic hyperfunction (1) and glutamatergic hypofunction (2), are widely accepted conceptual frameworks for understanding the pathophysiology of schizophrenia and for the development of drugs for the treatment of either dopamine-related or glutamate-related symptoms of the disease $(2,3)$. This study addresses a third hypothesis, the "adenosine hypothesis of schizophrenia," which has recently been proposed as a novel concept to integrate the dopaminergic hyperfunction and glutamatergic hypofunction hypotheses (4).

The purine ribonucleoside adenosine modulates neurotransmission through activation of 4 types of $G$ protein-coupled adenosine receptors (ARs), $A_{1} R, A_{2 A} R, A_{2 B} R$, and $A_{3} R$, which exert spatially distinct functions within the brain on presynaptic and postsynaptic sites $(5,6)$. Presynaptically, adenosine regulates the release of both dopamine and glutamate $(7,8)$, whereas the output of dopaminergic and glutamatergic neurotransmission is regulated by heterodimerization of ARs with dopamine and glutamate receptors $(9,10)$. Through these mechanisms adenosine exerts upstream control over both dopaminergic and glutamatergic signaling. Consequently, any disruption in adenosine homeostasis is expected to affect those 2 transmitter systems, which play fundamental roles in the pathophysiology of schizophrenia. This regulatory function of adenosine might provide a missing link for the functional integration of the dopamine and glutamate hypotheses. Conventional antipsychotic

Authorship note: Hai-Ying Shen and Philipp Singer contributed equally to this work. Conflict of interest: The authors have declared that no conflict of interest exists. Citation for this article: J Clin Invest. 2012;122(7):2567-2577. doi:10.1172/JCI62378 drugs were not designed to modulate the dopaminergic and glutamatergic systems concomitantly, which might be needed to achieve more efficient treatment of the positive, negative, and cognitive symptoms of schizophrenia; however, manipulating adenosine as an upstream regulator of both dopamine and glutamate might offer such a concomitant treatment approach. A link between adenosine hypofunction and schizophrenia is also supported by clinical evidence: increased enzymatic degradation of adenosine by adenosine deaminase has been detected in patients with schizophrenia $(11,12)$, whereas allopurinol, a purine degradation inhibitor that increases adenosine tone, has shown clinical efficacy as add-on therapy for schizophrenia $(13,14)$.

Several lines of evidence indicate that hypofunction of adenosine might be closely linked to the dopaminergic abnormalities found in patients with schizophrenia, including elevated occupancy of dopamine $\mathrm{D}_{2}$ receptor $\left(\mathrm{D}_{2} \mathrm{R}\right)$ by dopamine, increased dopamine turnover, and enhanced amphetamine-induced dopamine release (4). Reduced adenosinergic signaling is expected to enhance basal dopaminergic activity by attenuating tonic inhibition of dopamine release as a result of reduced activation of presynaptic ARs (15). Within the striatum, adenosine deficiency should stimulate dopaminergic signaling via antagonistic interactions between $A_{1} R s$ and dopamine $D_{1}$ receptors $\left(D_{1} R s\right)$ in striatonigral neurons and between $D_{2}$ Rs and $A_{2 A}$ Rs in striatopallidal neurons $(16,17)$. These antagonistic AR-DR interactions in the striatum are also thought to underlie the motor-depressant and motor-stimulant effects of AR agonists and antagonists, respectively, and would predict a typical antipsychotic-like profile of $\operatorname{AR}$ agonism $(4,18)$.

Therapeutically, the systemic use of AR agonists is limited by severe cardiovascular and immunomodulatory side effects (6). A therapeutic alternative might be the direct modulation of the ambient level of adenosine, and this can be achieved by targeting 
A

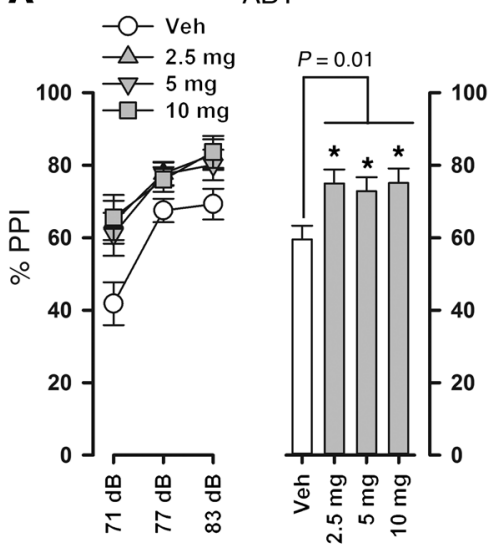

B ABT/APO

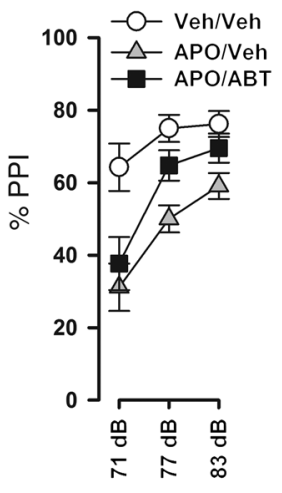

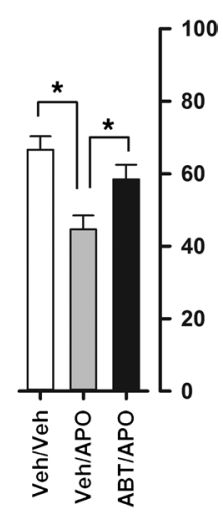

Figure 1

Antipsychotic-like activity of the ADK inhibitor ABT-702 in a PPI paradigm. PPI is indexed by percentage PPI (\%PPI), calculated by the following formula: ([pulse-alone] - [prepulse-plus-pulse]/[pulse-alone] × 100\%). Because ABT-702 (ABT) depressed baseline startle reaction to the 100 and $110 \mathrm{~dB}$ pulse stimuli but not to the $120 \mathrm{~dB}$ pulse stimulus, PPI was calculated based only on the $120 \mathrm{~dB}$ pulse condition in which the PPI data were not confounded by group differences on startle reaction. Percentage PPI data are shown as a function of prepulse intensity (left panels) as well as averaged over the 3 levels of prepulse (right panels). (A) ABT-702 enhanced basal PPI in drug-naive animals. This effect was independent of dose, as all of the tested doses led to similar enhancement in percentage PPI (0 mg/kg ABT-702, $n=10 ; 2.5 \mathrm{mg} / \mathrm{kg}$ ABT-702, $n=10$; $5 \mathrm{mg} / \mathrm{kg} \mathrm{ABT-702,} n=10 ; 10 \mathrm{mg} / \mathrm{kg} \mathrm{ABT-702,} n=9$ ). (B) Pretreatment with $5 \mathrm{mg} / \mathrm{kg}$ ABT-702 was also effective in antagonizing the PPI-disruptive effect of $2 \mathrm{mg} / \mathrm{kg}$ apomorphine (APO) (Veh/Veh, $n=13$; APO/Veh, $n=12$; APO/ABT, $n=11) .{ }^{*} P<0.05$, compared with the control group (Veh/Veh), Fisher's LSD post-hoc comparison, following a significant main effect of drug treatment in the overall ANOVA. Data are mean \pm SEM.

enzymes or nucleoside transporters that control the extracellular levels of adenosine (19). A promising target is adenosine kinase (ADK), the key enzyme of the metabolic adenosine clearance pathway (20). In the adult brain, ADK is predominantly expressed in astrocytes, in which it phosphorylates adenosine to AMP and thus drives the metabolic reuptake of adenosine through equilibrative nucleoside transporters (20).

To ameliorate confounding effects that might be caused by dynamic expression changes of the endogenous Adk gene (20-22), we recently developed a mutant mouse model (Adk-tg mice) with reduced adenosine tone in brain by replacing the endogenous $A d k$ gene with a loxP-flanked, constitutively expressed $A d k$ transgene that is overexpressed in brain and results in brain-wide adenosine hypofunction (22-24). This genetic manipulation produces behavioral phenotypes considered relevant to schizophrenia, including working memory deficit, enhanced novelty induced hyperactivity, and abnormal responses to psychomimetic drugs (25). This animal model supports our central hypothesis that adenosine hypofunction, and thereby disruption of adenosine homeostasis, can give rise to schizophrenia-like behavioral abnormalities.

In this study, we provide the first preclinical evidence to our knowledge that systemic pharmacological inhibition of ADK possesses antipsychotic-like activity in normal animals and demonstrate the presence of attentional impairments closely linked to schizophrenia in Adk-tg mice, thus supporting the basic adenosine hypofunction hypothesis of schizophrenia and the antipsychotic potential of adenosine augmentation therapy. In mechanistic studies, we (a) measure of sensorimotor gating deficits believed to underlie sensory flooding and cognitive fragmentation in schizophrenia (28, 29). PPI disruption induced by the dopaminergic agonist apomorphine in rodents is a well-established animal model of schizophrenia, with predictive validity for antipsychotic drugs (30). In addition, the enhancement of basal PPI has been proposed as a marker of antipsychotic action, since antipsychotics increase PPI in drugnaive animals (31). We therefore tested the efficacy of ABT-702 to (a) increase PPI in naive WT C57BL/6 mice and to (b) reverse apomorphine-induced PPI disruption. A dose-response analysis $(0,2.5,5$, and $10 \mathrm{mg} / \mathrm{kg}, n=9-10$ per dose $)$ revealed that ABT702 increased PPI independent of dose $\left(\mathrm{F}_{3,36}=3.591, P=0.023\right)$, a finding that was confirmed by subsequent Fisher's least significant difference (LSD) post-hoc comparisons (Figure 1A). Furthermore, ABT-702 was effective in reversing the PPI-disruptive effect of apomorphine $(2 \mathrm{mg} / \mathrm{kg})$. As expected, the acute challenge with apomorphine (treatment with vehicle and apomorphine [Veh/ APO], $n=12$ ) strongly attenuated PPI relative to that in vehicletreated animals (treatment with vehicle alone [Veh/Veh], $n=13$ ), an impairment that was reversed by pretreatment with $5 \mathrm{mg} / \mathrm{kg}$ ABT-702 (treatment with ABT-702 and apomorphine [ABT/ APO], $n=11$ ), yielding a significant effect of drug treatment $\left(\mathrm{F}_{2,33}=8.699, P=0.001\right)$ (Figure $\left.1 \mathrm{~B}\right)$. Subsequent Fisher's LSD posthoc comparisons verified the significant reduction in percentage PPI in the Veh/APO group relative to that in both the Veh/Veh $(P=0.003)$ and $\mathrm{ABT} / \mathrm{APO}(P=0.004)$ groups. In both PPI experiments, ABT-702 was found to depress the startle reactivity on 


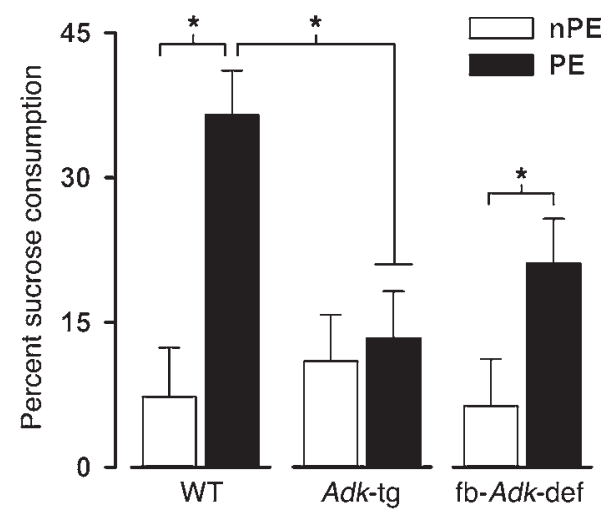

pulse-alone trials at pulse intensities of 100 and $100 \mathrm{~dB}_{\mathrm{A}}$ but not at $120 \mathrm{~dB}_{\mathrm{A}}$ (data not shown). Given that group differences on startle reactivity seriously confound the assessment of PPI, we restricted the analysis of PPI to the $120 \mathrm{~dB}_{\mathrm{A}}$ pulse condition, in which the startle reactivity on pulse-alone trials was highly comparable among groups. The PPI data showed for the first time to our knowledge that ADK inhibition enhanced basal PPI in WT C57BL/ 6 mice and exerted antipsychotic-like efficacy in a pharmacologically induced animal model of schizophrenia.

Adenosine-deficient Adk-tg mice show attentional deficits closely linked to schizophrenia. To evaluate whether adenosine deficiency might lead to schizophrenia-like phenotypes, we developed the Adk-tg mouse $(22,25)$ as a model of brain-wide adenosine deficiency (24). Adk-tg mice ubiquitously overexpress a constitutively expressed Adk transgene as replacement of the endogenous $A d k$ gene, which is subject to dynamic expression changes $(21,32)$. Previous behavioral assessment of the Adk-tg mice revealed a number of behavioral alterations relevant for schizophrenia, such as spontaneous locomotor hyperactivity, working memory deficiency, and abnormal response to psychostimulants $(4,25)$. Since PPI could not be measured in the Adk-tg mice because these animals are deaf, we tested them for latent inhibition (LI), which refers to the phenomenon that non-reinforced preexposure of a neutral stimulus prior to conditioning is less effective in generating a conditioned response compared with a novel stimulus (33). It reflects the ability to ignore irrelevant stimuli and represents a form of selective attention that is impaired in patients with schizophrenia (33). Disruption of LI by amphetamine is a well-established animal model of schizophrenia with construct, face, and predictive validity (34). Here, LI was assessed in a conditioned taste aversion (CTA) paradigm, in which a single pairing of a neutral taste (sucrose) with sickness (gastric malaise induced by $\mathrm{LiCl}$ injection) produces a strong aversion to that taste. Inconsequential preexposure of sucrose prior to its pairing with sickness attenuates the strength of the taste aversion in WT mice, which constitutes the LI effect (35). CTA was assessed in a 2-bottle choice test 24 hours after conditioning, in which the animals could freely choose between sucrose solution and water. The strength of the aversion was indexed by the amount of sucrose consumption, expressed as percentage of total liquid intake (sucrose solution and water). Thus, the lower the value, the stronger the aversion. As shown in Figure 2, LI was clearly present in WT mice, as indicated by increased sucrose intake in preexposed (PE) animals compared with that in non-PE (nPE) animals. By contrast, LI was completely abolished in Adk-tg mice characterized by brainwide adenosine deficiency. To assess whether this LI phenotype

\section{Figure 2}

$\mathrm{LI}$ in the CTA paradigm. Taste aversion was indexed by percentage sucrose consumption. The lower the value, the greater the taste aversion. Weaker aversion in PE animals relative to that in $\mathrm{nPE}$ animals constitutes the LI effect. LI was present in WT and fb-Adk-def mice but completely abolished in Adk-tg mice ( $n=9-11$ per group). ${ }^{*} P<0.05$ based on post-hoc comparison, following a significant interaction in the overall ANOVA. Data are mean \pm SEM.

was modifiable by increasing cortical/hippocampal adenosine, we included littermates with Emx1-Cre-mediated deletion of the floxed Adk-tg transgene (fb-Adk-def littermates), which are characterized by reduced ADK expression in cortex and hippocampus and a 1.63-fold increase in adenosine compared with that in WT animals (24). LI reemerged in fb-Adk-def mice, indicating that local augmentation of cortical/hippocampal adenosine can at least partially restore the LI disruption induced by global adenosine deficiency. These impressions were supported statistically by a $2 \times 3$ (preexposure $\times$ genotype) ANOVA $(n=9-11$ per group) revealing a significant preexposure effect $\left(\mathrm{F}_{1,55}=15.48, P<0.001\right)$ as well as the critical preexposure $\times$ genotype interaction $\left(F_{2,55}=3.83\right.$, $P=0.028)$. Subsequent Fisher's LSD post-hoc comparisons confirmed the presence of LI in controls (WT nPE vs. WT PE, $P<0.001$ ) and $\mathrm{fb}$ - $A d k$-def mice (fb- $A d k$-def nPE vs. fb-Adk-def PE, $P=0.048$ ) but not in $A d k$-tg mice ( $A d k$-tg nPE vs. $A d k$-tg PE, $P=0.728$ ). Importantly, the LI phenotypes in the mutant mice resulted primarily from increased aversion in the PE conditioning and not from a deficit in fear conditioning as such (in nPE animals). This was confirmed by a significant genotype effect $\left(\mathrm{F}_{2,29}=4.71, P=0.021\right)$ when the ANOVA was restricted to the PE condition.

Hence, the complete abolition of LI in Adk-tg mice indicates that a global decrease in the adenosine tone can induce attentional deficits closely linked to schizophrenia. We believe that this is the first demonstration of a schizophrenia-like phenotype in Adk-tg mice in a standard animal model of schizophrenia and that this thus provides further credence to the adenosine hypofunction hypothesis of schizophrenia. The LI effect reemerged in $\mathrm{fb}$-Adkdef mice, demonstrating that regional augmentation of adenosine via telencephalon-specific deletion of ADK was able to reinstate LI in the Adk-tg genetic background of global ADK overexpression. However, LI was still weaker than that in WT mice, indicating that LI expression was not fully normalized in fb-Adk-def mice.

$A_{2 A}$ receptor activation restores responsiveness to amphetamine in adenosine-deficient mice. Our previous data revealed a strong attenuation in the psychomotor response to amphetamine in the Adk-tg mice, indicating a direct mechanistic link between the adenosine and dopamine systems $(4,25)$. The following experiments were designed to reveal the mechanistic contribution of $A_{1} R$ and $A_{2 A} R$ to the amphetamine phenotype. To this end, we quantified amphetamine-induced psychomotor activity in WT and Adk-tg mice as the distance traveled in the open-field paradigm and evaluated the impact of pretreatments with either the $\mathrm{A}_{1} \mathrm{R}$-specific agonist CCPA or the $\mathrm{A}_{2 \mathrm{~A}} \mathrm{R}$-specific agonist CGS-21680. As expected, amphetamine $(2.5 \mathrm{mg} / \mathrm{kg}$, i.p.) produced robust hyperlocomotion in WT 

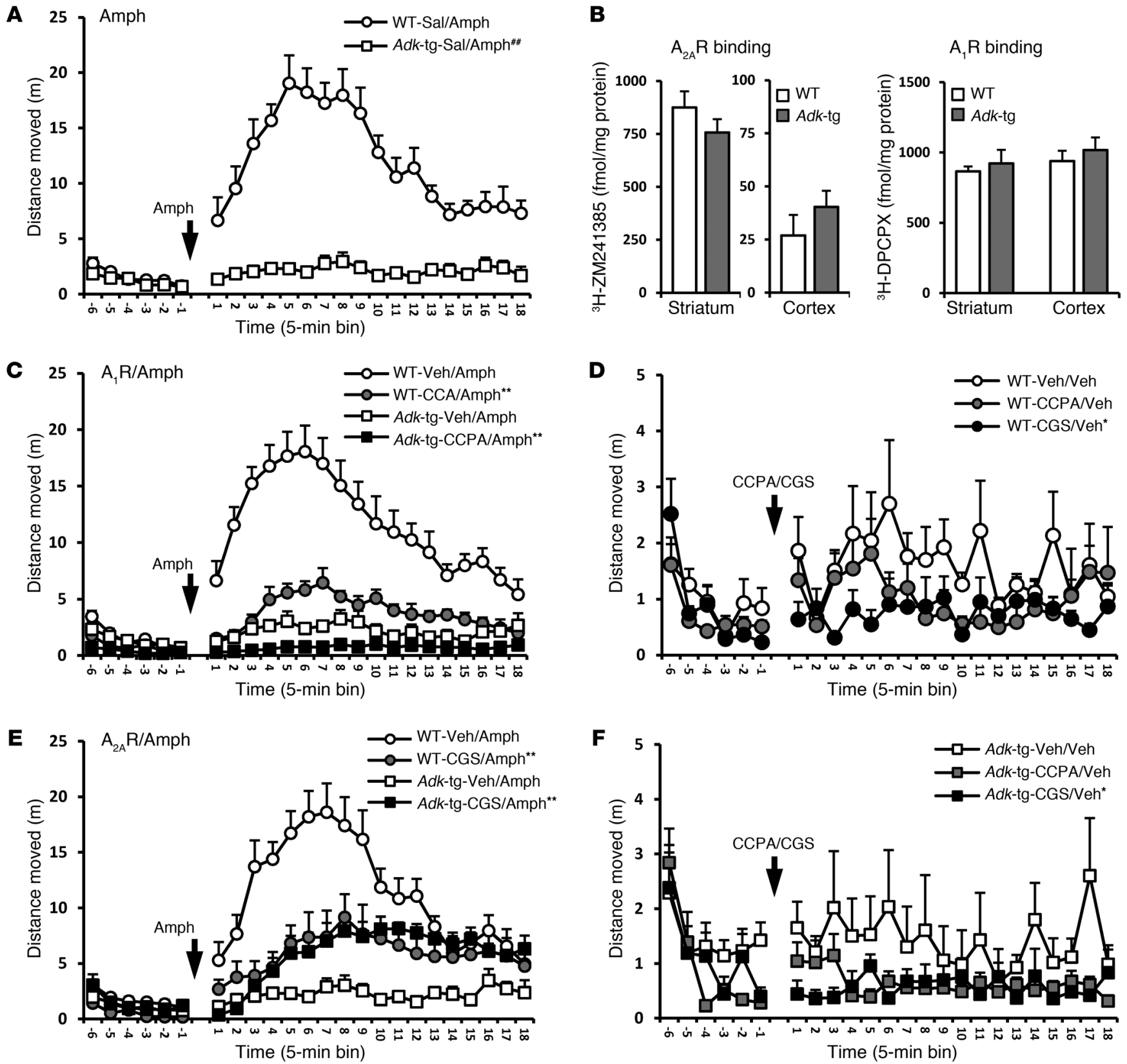

Figure 3

$\mathrm{A}_{2 \mathrm{~A}} \mathrm{R}$ mechanistically links ADK expression with psychomotor activity. (A and $\mathbf{C}-\mathbf{F}$ ) Psychomotor response to amphetamine (Amph; $2.5 \mathrm{mg} / \mathrm{kg}$, i.p.), in combination with selective AR agonists, was assessed in the open field and indexed as distance traveled. (A) Amphetamine-induced hyperlocomotion, as seen in WT mice following saline (Sal) treatment, is abrogated in Adk-tg mice $(n=10-12$ per genotype, $\mathrm{F}=45.545$, $P<0.01)$. (B) Membrane binding assay showed no significant change in binding densities of $A_{2 A} R\left({ }^{3} H-Z M 241385\right)$ and $A_{1} R\left({ }^{3} H-D P C P X\right)$ on whole-membrane preparations from striatum or cortex, comparing expression levels of untreated naive Adk-tg and WT mice ( $n=7-15 / g e n o t y p e /$ region, $\mathrm{F}$ values $=0.330-1.432, P>0.05$ ). (C) $A_{1} R$ agonist pretreatment (CCPA, $0.25 \mathrm{mg} / \mathrm{kg}$, i.p.) attenuated amphetamine-induced psychomotor activity in both WT and Adk-tg mice ( $n=8-9 /$ genotype/treatment, $F=48.212, P<0.01)$. (E) $A_{2 A} R$ agonist pretreatment (CGS-21680 [CGS], $0.5 \mathrm{mg} / \mathrm{kg}$, i.p.) attenuated amphetamine-induced psychomotor activity in WT mice $(n=8$ per group, $P<0.01)$; conversely, it potentiated the amphetamine effect in Adk-tg mice ( $n=8-9, F=13.358, P<0.01)$. ( $D$ and $\mathbf{F})$ CCPA injection alone did not affect motor activity in both genotypes ( $n=7-8$ per, $P>0.05$ ), while CGS-21680 alone slightly but significantly attenuated basal locomotion $(n=8-12$ per genotype, $P<0.05)$; this effect was comparable in Adk-tg and WT mice. Data are mean \pm SEM. $\# \#<<0.01$, versus WT with same treatment; ${ }^{*} P<0.05,{ }^{* \star} P<0.01$ versus vehicle (Veh) pretreatment within the corresponding genotypes, based on 2-way ANOVA. Arrows indicate injection time points. 
mice but failed to elicit any measurable motor response in $A d k$-tg mice $\left(n=10-12\right.$ per genotype, $F_{2,19}=45.545, P=0.01$; Figure $\left.3 \mathrm{~A}\right)$, validating our previous finding. Pretreatment with CCPA $(0.25 \mathrm{mg} /$ kg, i.p.) suppressed psychomotor response to amphetamine in both genotypes ( $n=8-9$ per genotype, $\mathrm{F}_{2,14}=48.212, P<0.01$; Figure $3 \mathrm{C}$ ) without affecting basal locomotor activity at the tested dose $(n=$ 8-12 per genotype, $P>0.05$; Figure $3, \mathrm{D}$ and $\mathrm{F}$ ). This outcome minimizes the involvement of $A_{1} R$ in the amphetamine phenotype of the Adk-tg mice. By contrast, when CGS-21680 (0.5 mg/kg, i.p.) was given prior to amphetamine, it significantly attenuated the motorstimulant effect of amphetamine in WT mice (compared with that after treatment with vehicle and amphetamine, $n=8-11, P<0.05$ ), but, strikingly, it potentiated the response to amphetamine in $A d k$-tg mice (compared with that after treatment with vehicle and amphetamine, $n=8-9, \mathrm{~F}_{2,14}=13.358, P<0.01$; Figure $\left.3 \mathrm{E}\right)$. Notably, the combined treatment of CGS-21680 and amphetamine resulted in similar activity levels in WT and Adk-tg mice, indicating that activation of the $\mathrm{A}_{2 \mathrm{~A}} \mathrm{R}$ was able to reverse amphetamine hyporesponsiveness in Adk-tg mice ( $n=8-9$ per genotype, $\mathrm{F}=0.706, P>0.05$; Figure $3 \mathrm{E})$. The obtained pattern of results cannot be explained by differential effects of CGS-21680 on baseline locomotor activity, because CGS-21680 treatment alone slightly depressed motor activity in both genotypes $(n=7-8$ per treatment, $P>0.05$; Figure $3 \mathrm{~F}$ ). Together, these results show that (a) abnormal psychomotor activity in Adk-tg mice is related to insufficient activation of $\mathrm{A}_{2 \mathrm{~A}} \mathrm{Rs}$ due to reduced endogenous adenosine and (b) that stimulation of $\mathrm{A}_{2 \mathrm{~A}} \mathrm{R}$ by CGS-21680 oppositely affects the psychomotor response to amphetamine in WT mice compared with that in Adk-tg mice (Figure 3E), suggesting that the effect of CGS-21680 might depend on the ambient level of endogenous adenosine. These findings provide a mechanistic link among adenosinergic tone, $\mathrm{A}_{2 \mathrm{~A}} \mathrm{R}$ activation, and dopaminergic stimulation.

Importantly, the attenuated response to amphetamine in Adk$\operatorname{tg}$ mice was not due to stereotypy, which would be indicative of a leftward shift of the dose-response curve, as the tested dose of amphetamine did not induce stereotypic behavior in either WT or Adk-tg mice. Furthermore, to rule out the contribution of intrinsic changes that are independent of the adenosine tone in Adk-tg mice, we performed a radioligand membrane binding assay to quantify $\mathrm{A}_{1} \mathrm{R}\left({ }^{3} \mathrm{H}\right.$-DPCPX) and $\mathrm{A}_{2 \mathrm{~A}} \mathrm{R}\left({ }^{3} \mathrm{H}-\mathrm{ZM} 241385\right)$ densities on wholemembrane preparations from different brain regions of drug-naive $A d k$-tg and WT mice. The quantitative analysis of ${ }^{3} \mathrm{H}-\mathrm{DPCPX}$ and ${ }^{3} \mathrm{H}-\mathrm{ZM} 241385$ binding densities revealed no significant differences of $A_{1} R$ and $A_{2 A} R$ expression levels between $A d k$-tg and WT mice in either the cerebral cortex or the striatum $(n=7-15$ per genotype per region, $\mathrm{F}$ values $=0.330-1.432, P>0.05$; Figure $3 \mathrm{~B}$ ). These findings suggest that $A d k$-tg mice have an intact repertoire of ARs and that any behavioral changes in these animals are the result of insufficient AR activation by endogenous adenosine.

Cell therapy to augment adenosine signaling in adenosine-deficient mice. Our data suggest that therapeutic adenosine augmentation might be a promising strategy for the treatment of schizophrenia. Given that adenosine exerts different functions in different brain regions $(5,6)$, it is imperative to identify the region-specific effects of adenosine that are relevant for schizophrenia. We therefore choose an adenosine augmenting cell therapy approach, based on baby hamster kidney (BHK) cells lacking ADK, to locally enhance adenosinergic signaling in discrete brain areas $(26,36)$. The BHKAK2 cell clone used here completely lacked ADK (Figure 4A), and, as previously shown (26), released about 20 ng adenosine per $10^{5}$ cells during the first hour of incubation (Figure 4B). These cells release adenosine in therapeutically effective doses, as intraventricular implants of BHK-AK2 cells almost completely suppressed seizures in a rat model of epilepsy via enhanced activation of the $A_{1} R$ by adenosine released from the cellular implant (26).

To evaluate the involvement of different brain region-specific adenosinergic mechanisms in the psychomotor and cognitive dysfunctions seen in Adk-tg mice, we designed a discrimination approach in which we implanted adenosine-releasing BHK-AK2 cells or corresponding WT BHK cells (BHK-WT cells) into either the hippocampus or striatum of WT or Adk-tg mice. Graft-recipients were tested 10 days following the cell implantation for either amphetamine-induced psychomotor response or for working memory. Animals were euthanized 3 weeks after the cell implantation, and brains were subjected to histological analysis (Figure 4, C and D). Nissl staining of coronal brain sections from tested mice localized the graft in the striatum (Figure $4 \mathrm{C}$, left) or above the CA1 pyramidal cell layer of the hippocampal formation (Figure 4D). In addition, brain sections subjected to ADK immunohistochemistry staining demonstrated differential ADK expression between BHK-WT and BHK-AK2 cell grafts compared with that in host cells in Adk-tg recipients with overexpressed ADK (Figure 4C). Importantly, while BHK-WT cell grafts with normal ADK expression showed prominent ADK immunoreactivity (Figure 4C), BHK-AK2 grafts appeared as an ADK-negative white cell mass (Figure $4 \mathrm{C}$ ). The graft location was consistent among treatment groups (Figure 4, E and F).

Adenosine-releasing intrastriatal cell grafts restore responsiveness to amphetamine in Adk-tg mice. To determine whether the focal cellbased release of adenosine in the striatum can affect responsiveness to amphetamine in Adk-tg mice, using mice with the graft in the hippocampus as the relevant controls, we subjected 4 groups of mice [(a) BHK-WT cell graft in striatum; (b) BHK-AK2 cell graft in striatum; (c) BHK-WT cell graft in hippocampus; and (d) BHK-AK2 cell graft in hippocampus; $n=9-12$ per group] to an amphetamine challenge $(2.5 \mathrm{mg} / \mathrm{kg}$, i.p.; Figure 5$)$ and quantified psychomotor activity by distance moved. Assessment of psychomotor activity was performed 10 days after cell implantation and demonstrated that neither intrastriatal implants of BHK-WT cells $(n=9)$ nor suprahippocampal implants of either cell type $(n=9-12$ per group, $P>0.05$ ) restored responsiveness to amphetamine (Figure 5, A and B). Importantly, only Adk-tg mice with intrastriatal BHK-AK2 grafts $(n=10)$ showed a significantly enhanced psychomotor response compared with Adk-tg mice receiving BHK-WT grafts $(n=8, P<0.01$; Figure $5 \mathrm{~A})$. These data demonstrate that cell-based reconstitution of adenosine signaling in striatum, but not in hippocampus, was sufficient to reinstate responsiveness to amphetamine in Adk-tg mice.

Adenosine-releasing suprahippocampal cell grafts reverse working memory deficits in Adk-tg mice. A similar cell graft approach was used to assess the contribution of the hippocampus and striatum to the impairment of cognitive functions in $A d k$-tg mice $(4,25)$. Here, we assessed working memory using a delayed-alternation task in the T-maze. In this task, the animals had to learn to alternate continuously between 2 possible choice arms in order to obtain a food reward. To make a correct choice on a given trial they had to recollect the response made on the previous trial, which requires the use of working memory. The magnitude of the baseline deficiency in Adk-tg mice was determined by a comparison between untreated Adk-tg mice $(n=10)$ and WT mice $(n=13)$. WT mice clearly performed better than chance $(50 \%)$ in correctly selecting the arm that 
A

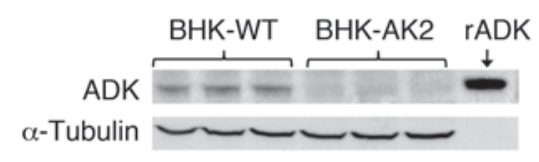

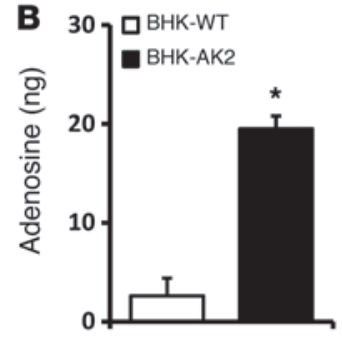
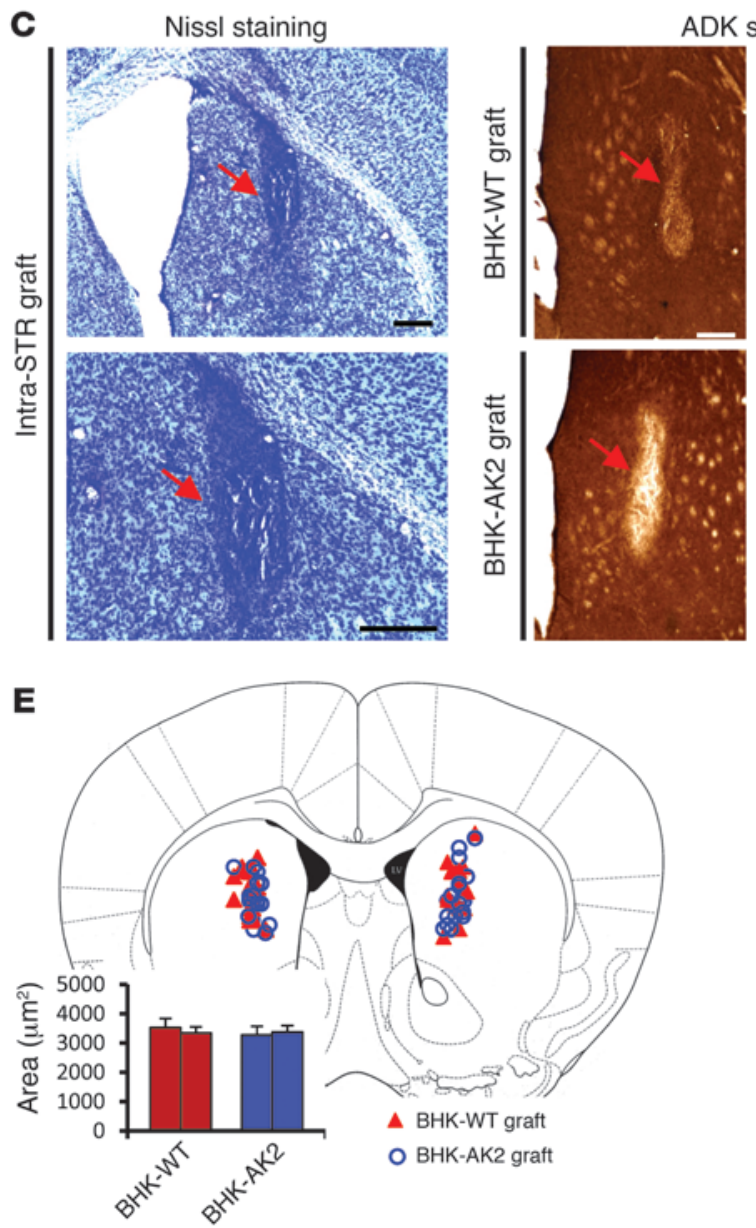
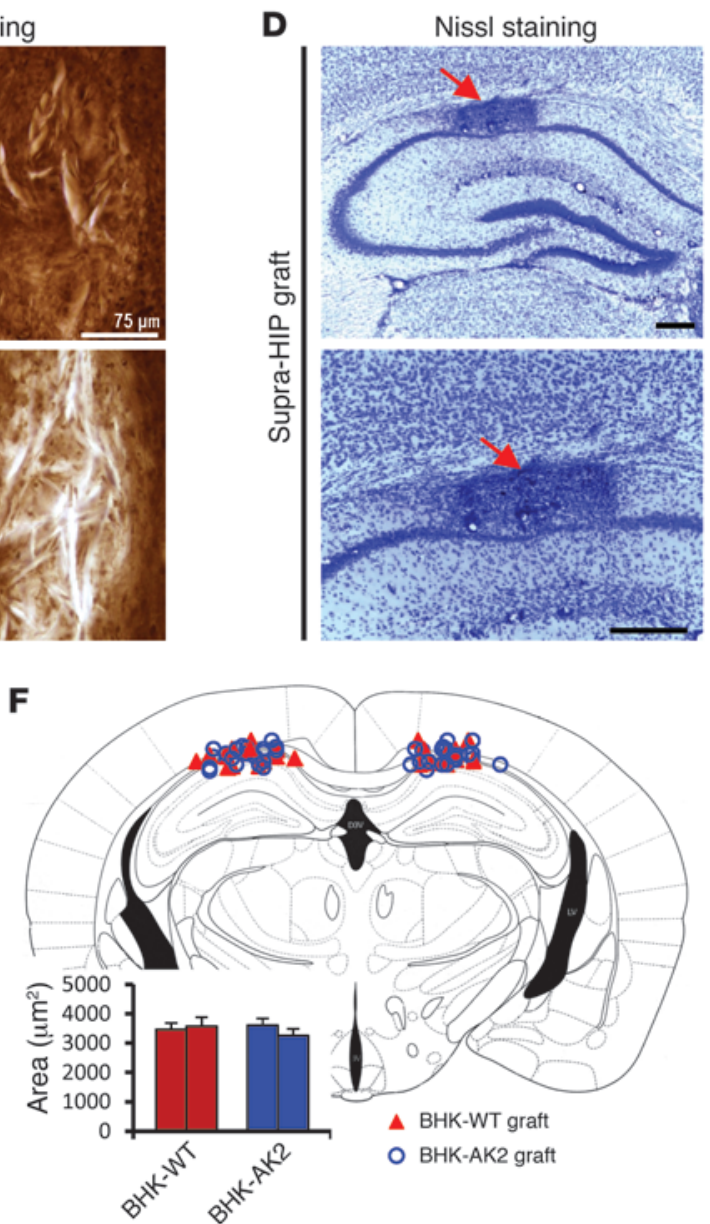

Figure 4

BHK cell-based adenosine augmentation approach. (A) Representative Western blot from cell extracts of BHK-AK2 (completely lacking ADK expression) and BHK-WT cells (with normal ADK expression, as control) that were used for cell transplantations. Anti-tubulin immunoreactivity was used to normalize for equal loading, and recombinant ADK (rADK) was loaded for comparison. (B) Demonstration of adenosine release from BHK-AK2 cells, which released about $20 \mathrm{ng}$ adenosine per $10^{5}$ cells during the first hour of incubation. (C and $\left.\mathbf{D}\right)$ Nissl staining of coronal brain sections from graft recipients 3 weeks after grafting into Adk-tg recipients validated the location of the graft (red arrows) in either (C, left) the striatum or (D) above the CA1 pyramidal cell layer of the hippocampal formation. ADK immunohistochemistry (C, right) demonstrated ADK expression in BHK-WT grafts (C, top right) but lack of ADK immunohistochemistry in BHK-AK2 grafts (C, bottom right). The locations of BHK-WT and BHK-AK2 cell grafts of each animal tested are shown superimposed on a standard mouse brain atlas image of the (E) striatum (AP, $1.00 \mathrm{~mm}$ from bregma) and (F) hippocampus (AP, $-2.10 \mathrm{~mm}$ ). The graphs by the atlas images indicate the average area of the corresponding cell grafts. Data are mean \pm SEM. ${ }^{\star} P<0.01$, versus BHK-WT. Scale bar: $300 \mu \mathrm{m}$.

was not entered on the previous trial, with a progressive increase in percentage of correct choices (68.9\% to $75.6 \%$ from test 1 to test 3 ). In contrast, $A d k$-tg mice performed close to chance level, with the percentage of correct choices ranging from $48.2 \%$ (test 1) to $59.1 \%$ (test 3 ), indicating a significant reduction in performance in com- parison with that of WT mice $(P<0.01$; Figure $6 \mathrm{~A})$. Next, we tested whether local cell-based adenosine augmentation would be capable of reversing the working memory deficiency in Adk-tg mice. To this end, we performed suprahippocampal or intrastriatal cell implantation of either BHK-WT or BHK-AK2 cells into naive Adk-tg 

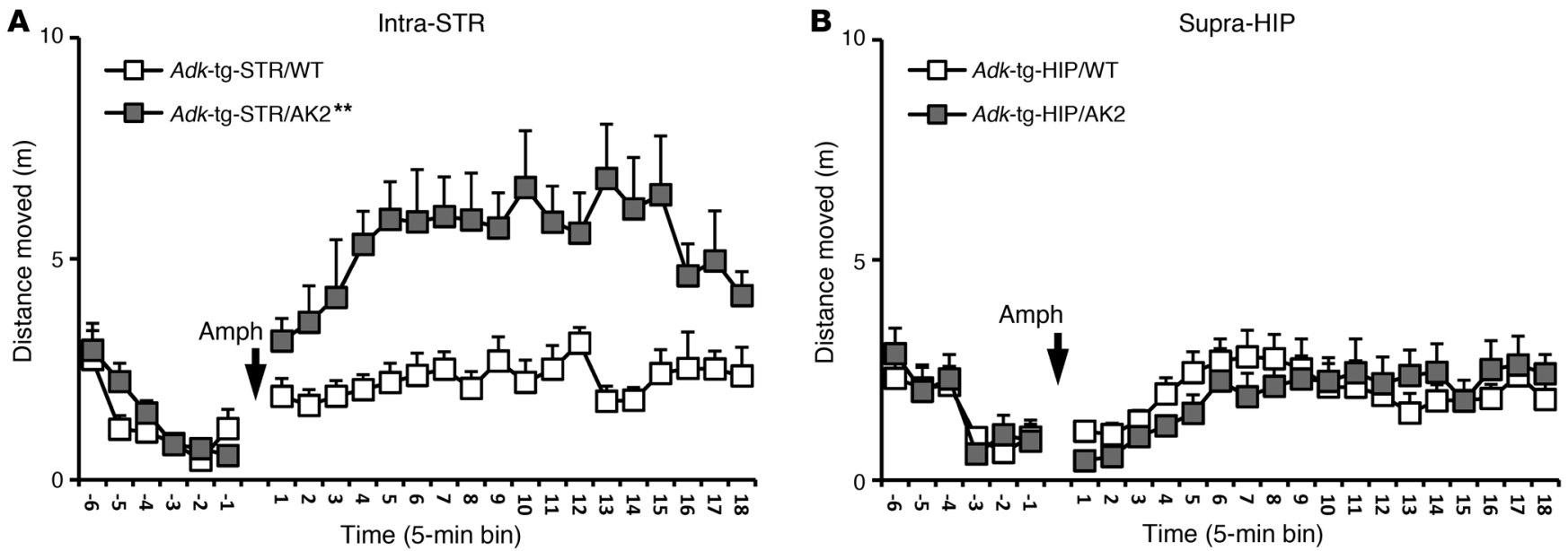

\section{Figure 5}

Intrastriatal adenosine augmentation restored responsiveness to amphetamine in Adk-tg mice. Psychomotor response to amphetamine (2.5 mg/kg, i.p.) in Adk-tg mice with bilateral intrastriatal (intra-STR) or suprahippocampal (supra-HIP) cell implantation with BHK-AK2 or BHKWT (control) cells was assessed in the open field and indexed as distance traveled. (A) Intrastriatal BHK-AK2 cells restored abnormal amphetamine response in Adk-tg mice compared with that in BHK-WT controls $(n=9-10$ per group, $P<0.01)$. (B) However, in Adk-tg mice, suprahippocampal BHK-AK2 cells showed no affect on the amphetamine-induced psychomotor response $(n=9-12$ per group, $P>0.05)$. Data are mean \pm SEM. ${ }^{* \star} P<0.01$, versus BHK-WT, 2-way ANOVA. Arrows indicate time points of amphetamine injection.

mice. Following 10 days of recovery from surgery, the animals were tested in the same T-maze delayed-alternation test of working memory. By the third test, suprahippocampal BHK-AK2 cell grafts significantly enhanced performance in Adk-tg mice $(n=10)$, while that of $A d k$-tg mice receiving BHK-WT cell grafts remained deficient $(n=8, P<0.01$; Figure $6 \mathrm{~B})$. Adenosine augmentation targeting the striatum was by contrast without any effect. Intrastriatal BHK-AK2 cell grafts were demonstrably effective in reinstating responsiveness to amphetamine but did not have any impact on T-maze performance; intrastriatal BHK-AK2 cell-grafted Adk-tg mice $(n=9)$ performed poorly, with $43.0 \%-55.0 \%$ correct choices, and were comparable to intrastriatal BHK-WT grafted Adk-tg mice $(n=8, P>0.05$; Figure $6 \mathrm{C})$ that also did not perform better than chance $(41.7 \%-45.0 \%$ correct choices). Hence, intrahippocampal but not intrastriatal adenosine-releasing cell grafts were able to reverse the working memory deficit in Adk-tg mice.

\section{Discussion}

Recent data suggest that hypofunction of adenosine might be implicated in schizophrenia, and restoration of adenosine function thus may offer novel therapeutic opportunities. This study evaluated the adenosine hypofunction hypothesis of schizophrenia in mice and identified the regional contributions of adenosine hypofunction to the genesis of schizophrenia-like behavioral abnormalities. Further, we revealed the mechanistic requirements for adenosine-based therapeutic interventions and present 2 promising adenosine augmentation strategies that possess antipsychotic potential: (a) pharmacological inhibition of ADK and (b) focal augmentation of adenosine by therapeutic brain implants of adenosine-releasing cells.

Systemic ADK inhibition exerts antipsychotic-like efficacy. PPI indicates a form of sensory gating that is consistently impaired in patients with schizophrenia and reliably disrupted by dopaminergic agonists in animals. Given that adenosine is an important modulator of dopaminergic activity, it is not surprising that it assumes a regulatory role in sensory gating $(4,37)$. In line with this assumption, the nonselective AR antagonist, theophylline $(6.6 \mathrm{mg} / \mathrm{kg})$, impaired sensory gating in healthy human volunteers. In rodents, genetic studies revealed that normal sensorimotor gating requires tonic activation of $\mathrm{A}_{2 \mathrm{~A}} \mathrm{Rs}$ (38). Here, we demonstrated that augmentation of adenosine tone achieved by systemic treatment with the ADK inhibitor ABT-702 (a) enhanced basal PPI in naive mice and (b) reversed apomorphine-induced PPI disruption (Figure 1). These effects resemble the profile of conventional antipsychotic drugs and together constitute the first demonstration to our knowledge of antipsychotic-like activity of an ADK inhibitor in animals. Our data thus provide preclinical proof of concept for adenosine augmentation therapy in schizophrenia.

Adk-tg mice - an effective mouse model of adenosine bypofunction in schizophrenia. To explore plausible causative links between adenosine dysfunction and selected schizophrenia-like phenotypes, an ADK-overexpressing mouse line has been generated $(22,25)$. The brain-wide constitutive transgenic overexpression of ADK decreases the ambient concentration of adenosine $(24,39)$ and produces abnormalities in multiple schizophrenia-relevant domains, including working memory, selective attention, novelty-induced locomotor activity, and sensitivity to psychostimulant drugs (25). These phenotypes are rather specific, given that the mutant mice are free from any abnormality in motor function, anxiety, social cognition, simple associative learning, or spatial memory. While Adk-tg and $\mathrm{fb}$-Adk-def mice were bred as littermates to directly attribute differences in phenotypes to telencephalon-specific changes in ADK expression, we included strain-, sex-, and age-matched C57BL/6 WT mice from different litters that were maintained under identical conditions as control. The choice of this breeding strategy as well as the use of constitutive mutants is a limitation of this study. However, several lines of evidence indicate that the effects of the targeted mutations of ADK are more profound than the impact of possible problems that might arise from using age- and sexmatched WT C57BL/ 6 mice, which were maintained under identi- 

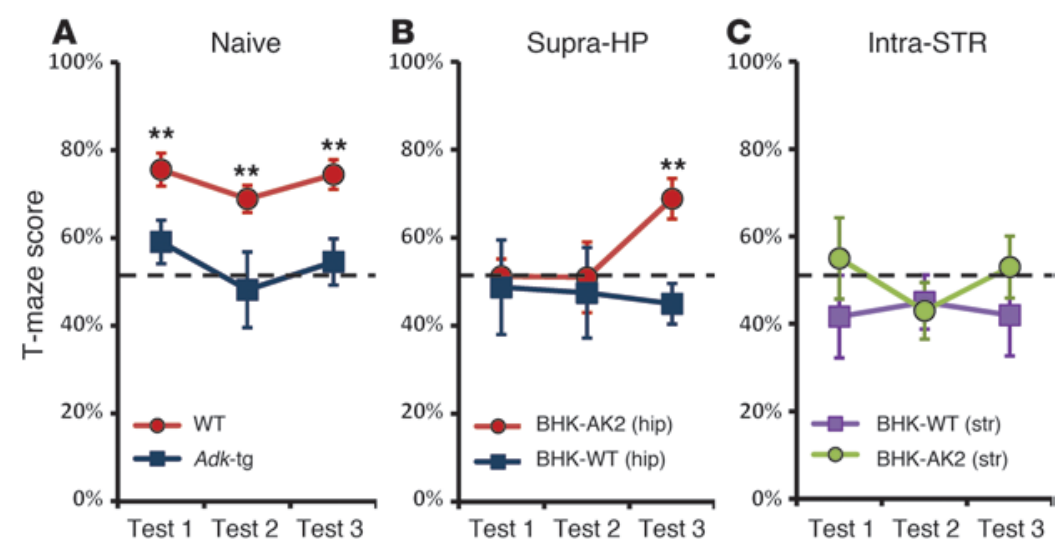

Figure 6

Adenosine-releasing suprahippocampal cell grafts reverse working memory deficits in Adk-tg mice. Working memory of Adk-tg and WT mice was tested using a delayedalternation task in the T-maze. (A) T-maze score of baseline working memory deficits in untreated Adk-tg mice $(n=10)$, with a lower percentage of correct choices $(48.2 \%-$ $59.1 \%)$ in test 1 through test 3 compared with that of WT mice $(n=13)(68.9 \%-75.6 \%$ correct choices). (B) Suprahippocampal BHK-AK2 cell implants significantly enhanced T-maze performance in Adk-tg mice $(n=10)$ at test 3, while performance of Adk-tg mice receiving BHK-WT cell grafts remained deficient $(n=8, P<0.01)$. (C) Adk-tg mice with intrastriatal BHK-AK2 cell grafts $(n=9)$ showed impaired working memory (43.0\%-55.0\% correct choices), similar to that of Adk-tg mice with BHK-WT grafts $(41.7 \%-45.0 \%$ correct choices) throughout tests 1 through $3(n=8, P>0.05)$. The dashed line denotes performance by chance $(50 \%)$. Data are mean $\pm \mathrm{SEM} .{ }^{*} P<0.01$, versus corresponding group within same test, 2-way ANOVA.

cal conditions. (a) The predicted changes in extracellular adenosine concentration and ADK expression in Adk-tg and fb-Adk-def mice relative to those in WT mice have been consistently validated multiple times, in different paradigms, and over several years (22, 24). (b) The present cell graft experiments demonstrated that local increases in extracellular adenosine altered the behavioral phenotypes of Adk-tg mice in the expected direction; this effect is not confounded by any potential background issues. (c) Expression levels of ARs were comparable between Adk-tg and WT mice (Figure 3), excluding the possibility that behavioral differences were due to changes in genotype-related differences in the number of ARs. (d) The critical behavioral phenotypes of Adk-tg mice, which have been studied for more than 7 years, were consistent across time, laboratories, and paradigms. The amphetamine phenotype described here replicates our previous findings from studies performed in a different laboratory (25) using Adk-tg mice from a different colony. Likewise, the working memory phenotype has now been demonstrated in 2 different laboratories using 2 different test paradigms - Morris water maze (25) and T-maze (present study). The consistency of a variety of critical phenotypes across different studies and laboratories $(22,24,25,32,39,40)$ lends further credence to the specificity of molecular links between genotype and phenotype of our mutants. Thus, the Adk-tg mouse line provides a valid animal model to study the effects of adenosine hypofunction within the context of schizophrenia and to evaluate therapeutic interventions designed to augment adenosine signaling.

Adenosinergic regulation of psychomotor behavior. Psychostimulantinduced augmentation of dopaminergic activity causes hyperlocomotion in rodents, a quantifiable response that is widely used experimentally to study susceptibility to psychotic symptoms (41). $\mathrm{D}_{1} \mathrm{Rs}$ and $\mathrm{D}_{2} \mathrm{Rs}$ on striatonigral and striatopallidal neurons, respectively, play crucial roles in the control of motor responses (42). Since $\mathrm{A}_{1} \mathrm{Rs}$ and $\mathrm{A}_{2 \mathrm{~A}} \mathrm{Rs}$ antagonistically interact with $\mathrm{D}_{1}$ Rs and $\mathrm{D}_{2}$ Rs, respectively $(10,43,44)$, adenosine is expected to exert a regulatory influence on amphetamine-induced psychomotor behavior. In line with these receptor-receptor interactions, reduced adenosinergic tone in Adk-tg mice is expected to exert a stimulatory influence on dopamine receptors, which is also considered the primary mechanism underlying the motorstimulant effect of the mixed $\mathrm{A}_{1} \mathrm{R} / \mathrm{A}_{2 \mathrm{~A}} \mathrm{R}$ antagonist caffeine (45). Consistent with this interpretation, $A d k$-tg mice were hyperactive in a novel environment (25) and showed impaired LI (Figure 2), a widely established model of schizophrenia closely linked to striatal hyperdopaminergia (46). Against this background, the strong reduction in the motor response to amphetamine detected in the Adk-tg mice (23) is an interesting finding; since the genetic $A d k$ manipulations used here are constitutive, we cannot rule out the contribution of developmental or compensatory factors to this phenomenon. In spite of this limitation, our data provide what we believe to be novel mechanistic insight into the regulation of amphetamine-induced psychomotor behavior by adenosine. We demonstrated an opposite impact of pharmacological $\mathrm{A}_{2 \mathrm{~A}} \mathrm{R}$ agonism on amphetamine-induced psychomotor stimulation in Adk-tg mice compared with that in WT mice. Namely, the $\mathrm{A}_{2 \mathrm{~A}} \mathrm{R}$-selective agonist, CGS-21680, potentiated the amphetamine response in the Adk-tg mice - respectively, reversed their hyporesponsiveness - but attenuated this psychomotor response in WT mice. The observed bidirectional regulatory manner indicates that pharmacological $\mathrm{A}_{2 \mathrm{~A}} \mathrm{R}$ agonism oppositely modulates dopamine-induced psychomotor stimulation under conditions of physiological or reduced adenosine tone: this may caution the proposed use of $\mathrm{A}_{2 \mathrm{~A}} \mathrm{R}$ agonists as antipsychotic treatment if adenosine levels are indeed reduced in patients with schizophrenia. By contrast, selective $A_{1} R$ activation by CCPA exerted a similar regulatory pattern, i.e., attenuation of amphetamine response in WT and Adk-tg mice. This finding indicates that the hyporesponsiveness to amphetamine seen in Adk-tg mice is attributed to an underactivation of the $A_{2 A} R$ rather than the $A_{1} R$. This is in keeping with a similar suppression of amphetamine-induced motor stimulation following genetic inactivation of the $\mathrm{A}_{2 \mathrm{~A}} \mathrm{R}$ (47). In addition, the hyperlocomotor response to amphetamine could be reinstated in Adk-tg mice by intrastriatal but not intrahippocampal adenosine delivery using adenosine-releasing cell grafts (Figure 5). Together, these findings indicate that $\mathrm{A}_{2 \mathrm{~A}} \mathrm{Rs}$ located in the striatum play a critical role in the regulation of amphetamine-induced psychomotor responses and demonstrate that manipulating the adenosine tone can affect dopamine-related behavior, which is of relevance for schizophrenia.

Adenosinergic modulation of cognitive function. A core cognitive deficit in schizophrenia is impairment in working memory, which is generally understood as the capacity to manipulate information transiently held in short-term memory required to perform complex cognitive tasks, such as problem solving, comprehension, or reasoning. Working memory performance was impaired in adenosine-deficient Adk-tg mice (25), which sharply contrasts 
with the working memory enhancement seen following genetic inactivation of $A_{2 A} R$ (48). However, the latter was mainly attributed to the loss of striatal $\mathrm{A}_{2 \mathrm{~A}} \mathrm{Rs}$ (48) and likely reflects a regionspecific mechanism at the subcortical level rather than an effect on the neural processes underlying learning and memory in the cortex and/or hippocampus. One might therefore speculate that hippocampal LTP would be negatively affected in the presence of reduced adenosine tone, leading in turn to impaired working memory performance in adenosine-deficient Adk-tg mice. Indeed, a hippocampus-specific mechanism is strongly supported by the fact that the working memory deficit was corrected by local augmentation of adenosine achieved by regional implants of adenosine-releasing BHK cells in the hippocampus but not the striatum.

Conversely, intrastriatal but not suprahippocampal adenosine augmentation was found to partially reverse the psychomotor impairment in the Adk-tg mice. The functional double dissociation between striatal and hippocampal adenosine indicates that global adenosine deficiency impairs amphetamine-induced motor stimulation and working memory by spatially distinct and functionally independent mechanisms between the striatum and hippocampus, respectively, which can be selectively targeted by localized adenosine delivery to the respective brain region. This, however, does not exclude the possibility that adenosinergic signaling in other brain regions might contribute to the regulation of these two behaviors.

Hence, focal adenosine augmentation (using cell therapy) may open a novel avenue to the treatment of schizophrenia symptoms by increasing the ambient tone of adenosine specifically in the affected brain region(s). This strategy would be expected to have limited direct impact on the remaining brain and thus the capability to avoid possible undesirable side effects associated with systemic AR agonist treatment. In a next step, the therapeutic potential of the local adenosine augmentation approach needs to be further evaluated in adenosine-independent animal models of schizophrenia such as chronic phencyclidine exposure, neurodevelopmental lesion, or linkage-identified genetic alterations.

Possible adenosine angmentation therapies for clinical use. A promising strategy to upregulate extracellular adenosine is to inhibit the enzymatic activity of ADK. This can be achieved by systemic treatment with existing ADK inhibitors that exert antipsychoticlike activity in animals as demonstrated in the PPI paradigm here and is expected to be applicable to humans. A dietary approach to downregulate ADK involves the use of a ketogenic diet (32), which is a high-fat, low-carbohydrate diet originally established to treat epilepsy in children (49). It has been linked to the cessation of persistent psychotic symptoms reported in one patient with schizophrenia (50), providing tentative support for a link between ADK inhibition and antipsychotic efficacy. An increase in the ambient tone of adenosine may also mimic the clinically beneficial effects of allopurinol and dipyridamole, which augment the extracellular pools of adenosine by inhibiting its degradation and reuptake, respectively (51). Hence, adenosine-metabolizing enzymes and nucleotide transporters offer multiple routes to achieve elevation of extracellular adenosine (4).

However, pharmacologic or systemic adenosine augmentation might not be a therapeutic option due to the short half-life (seconds) and poor blood-brain barrier permeability of adenosine and/or undesirable side effects concerning cardiovascular and liver function (6). Likewise, systemic AR agonist treatment is limited by the sedative properties of $\mathrm{A}_{1}$-selective agonists and by immune- related and vascular side effects of $\mathrm{A}_{2 \mathrm{~A}}$-selective agonists (6). Furthermore, AR agonists generally show antipsychotic-like activity in classical hyperdopamine and hypoglutamate animal models of schizophrenia but also impair cognitive performance in normal animals (4). These differential and sometimes controversial effects of adenosine and AR agonists are indicative of the multiple regionspecific effects of adenosine and its receptors $(5,6)$. Thus, local increases in adenosine might minimize the risk of side effects of systemic AR agonism or adenosine administration, and this might be achieved by localized adenosine delivery using a cell therapy. Although BHK cells used here are not suitable for clinical translation and further preclinical studies are needed to assess the efficiency and safety of this approach, promising alternative strategies are currently being tested, such as local brain implants of silk polymers or stems cells engineered to release adenosine $(52,53)$. The present data further suggest that local augmentation of adenosine in the hippocampus may specifically ameliorate the cognitive symptoms in schizophrenia whereas focal adenosine delivery to the striatum may instead target psychomotor-related symptoms of the disease. Thus, symptom-specific treatment achieved by regionspecific adenosine augmentation therapy might be beneficial as add-on therapy to conventional antipsychotic mediation. Schizophrenia is a complex brain disease involving dysfunction of multiple brain circuits, and region-specific modulation of adenosine function may offer additional or supplemental pharmacological options for the treatment of schizophrenia.

\section{Methods}

Animals. Adk-tg and fb-Adk-def mice were generated as littermates and maintained on a C57BL/6 genetic background as described elsewhere (23). Briefly, double-transgenic $A d k$-tg mice are homozygous for a ubiquitously expressed loxP-flanked $A d k$ transgene ( $A d k-\operatorname{tg}$ ) on top of a homozygous $A d k$-null (Adk ${ }^{\mathrm{tm} 1 \mathrm{Bois}}$ ) background, whereas the triple-transgenic fb-Adk-def mice are heterozygous for an additional Emx1-Cre transgene, which drives dorsal telencephalon-specific deletion of the Adk transgene. Breeding Adk$\operatorname{tg}$ mice with $\mathrm{fb}$ - $A d k$-def mice generated $A d k$-tg mice and $\mathrm{fb}$ - $A d k$-def mice as true littermates in a 1:1 ratio (with the presence of the heterozygous Emx1Cre gene as the only genetic between-subjects factor). As a limitation of this study, the breeding of mutant and WT control animals as true littermates was not feasible due to high costs and a low frequency of obtaining all desired genotypes in the same litter. To mitigate potentially confounding issues, age- and sex-matched C57BL/6 WT mice, which were maintained in the same colony room and habituated under identical conditions, served as controls. The animals were housed in temperature- and humidity-controlled rooms, with a 12-hour-light/dark cycle. They were 10 to 12 weeks old at the beginning of behavioral testing and weighed 25 to $30 \mathrm{~g}$.

Drug treatments and locomotor assessments. Before drug treatment and locomotor assessment, all mice were habituated in the testing environment. Mice were i.p. administrated amphetamine $(2.5 \mathrm{mg} / \mathrm{kg})$, CGS$21680(0.5 \mathrm{mg} / \mathrm{kg})$, or CCPA $(0.25 \mathrm{mg} / \mathrm{kg})$, either separately or as combined treatment. In the combined treatment, CCPA or CGS-21680 was dissolved in vehicle, $5 \%$ DMSO and $95 \% \mathrm{dd}_{2} \mathrm{O}$, and injected 30 minutes before amphetamine (Figure 3). Psychomotor activity was indexed by distance traveled recorded in consecutive 5-minute bins as previously described (25). Data were acquired and processed by the Ethovison (Noldus Technology) tracking system.

Intrastriatal and suprahippocampal implantation of adenosine-releasing cells. To augment local adenosine levels, adenosine-releasing BHK-AK2 cells (26) or corresponding control BHK-WT cells were bilaterally transplanted into either striatum or above the CA1 layer of the hippocampal forma- 
tion of Adk-tg mice. Under general anesthesia (1.5\% isoflurane, $30 \% \mathrm{O}_{2}$, and $68.5 \% \mathrm{~N}_{2} \mathrm{O}$ ), mice received bilateral cell transplantation using a $5-\mu \mathrm{l}$ Hamilton syringe with a 34-gauge stainless steel injector (Plastics One). 50,000 cells were injected per hemisphere in an injection volume of $1.5 \mu \mathrm{l}$ at a rate of $0.5 \mu \mathrm{l}$ per minute. After injection, the needle was left in place for an additional 3 minutes to minimize reflux. Stereotactic procedures were used with coordinates of intrastriatal (anterior-posterior [AP], 1.00 $\mathrm{mm}$; medial-lateral, $\pm 1.60 \mathrm{~mm}$; dorsal-ventral, $-3.40 \mathrm{~mm}$ ) and suprahippocampal (AP, $-2.10 \mathrm{~mm}$; medial-lateral, $\pm 1.50 \mathrm{~mm}$; dorsal-ventral, -1.00 $\mathrm{mm}$ ) locations. All experiments were performed under standard immunosuppression: from 2 days prior to cell implantation all mice received daily injections of cyclosporine A (Sandimmune, Novartis, $15 \mathrm{mg} / \mathrm{kg}$, i.p.) throughout the entire period of experimentation. Ten days after cell implantation, the animals were subjected to assigned tests, followed by histological analysis 3 weeks later.

Immunohistochemistry and Nissl staining. To validate graft location after completion of the experiments, $A d k$-tg graft recipients were subjected to standard histological analysis. Brains were processed for Nissl (Cresyl Violet) staining as well as ADK immunohistochemistry as previously described (22). The location of cell grafts of each animal tested was superimposed on a standard mouse brain atlas image of the typical levels of striatum (AP, $1.00 \mathrm{~mm}$ from bregma) and hippocampus (AP, $-2.10 \mathrm{~mm}$ ). The area of each cell graft, at the level of striatum or hippocampus, was quantified in 3 adjacent histological sections from each animal.

Western blot analysis. To quantify ADK expression and adenosine release, BHK-WT and BHK-AK2 cells were cultured in Dulbecco's Modified Eagle Media (supplemented with $10 \% \mathrm{FBS}$ ). Adenosine release was quantified after culturing $10^{5}$ cells for 1 hour in fresh medium (26). Subsequently, cells were harvested for aqueous protein extraction. Twenty-five $\mu \mathrm{g}$ protein was separated by standard gel electrophoresis, transferred, and incubated overnight with primary ADK antibody $(1: 5,000)$. To normalize ADK immunoreactivity with protein loading, a mouse monoclonal anti- $\alpha$-tubulin antibody (sc-8035, Santa Cruz Biotechnology Inc.; 1:5,000) was used to reprobe the same blot, and the optical density ratio of ADK to $\alpha$-tubulin was calculated.

Radioligand membrane binding assays. Whole-membrane preparations were prepared from striatum and cortex of adult $A d k$-tg mice. Single-point saturation binding assays were performed in duplicate to quantify total $A_{1} R$ and $A_{2 A} R$ levels as described before (18). The radioligands and final concentrations used were as follows: (a) $\mathrm{A}_{1} \mathrm{R},{ }^{3} \mathrm{H}$-DPCPX ( $2 \mathrm{nM}$, specific activity 111.6 Ci/mmol; GE Healthcare); (b) $\mathrm{A}_{2 \mathrm{~A}} \mathrm{R},{ }^{3} \mathrm{H}-\mathrm{ZM} 241385$ (3 nM, specific activity $73.1 \mathrm{Ci} / \mathrm{mmol}$; PerkinElmer). Nonspecific binding was determined using $2 \mu \mathrm{M}$ xanthine amine congener (Sigma-Aldrich).

PPI. The apparatus and procedures were identical to those described previously (54). In brief, a PPI testing session comprised 10 series of discrete trials, with each series consisting of 3 pulse-alone trials (100, 110, and 120 $\left.\mathrm{dB}_{\mathrm{A}}\right), 3$ prepulse-alone trials $\left(71,77\right.$, or $\left.83 \mathrm{~dB}_{\mathrm{A}}\right)$, the 9 possible combinations of prepulse-plus-pulse trials, and 1 no-stimulus trial (constant $65 \mathrm{~dB}_{\mathrm{A}}$ background noise alone). PPI was indexed by percentage PPI, calculated by the following formula: ([pulse-alone] - [prepulse-plus-pulse]/[pulse-alone] $\times 100 \%$ ). In addition, the pule-alone trials were separately analyzed to measure the startle reaction. These data were first subjected to a logarithmic transformation $(\ln [$ reactivity score $+e])$ in order to improve data distribution and variance homogeneity (55). To disrupt PPI by apomorphine, the drug was administered subcutaneously 5 minutes before testing in a volume of $5 \mathrm{mg} / \mathrm{kg}$ body weight. It was dissolved in $1 \%$ ascorbic acid solution with the $\mathrm{pH}$ adjusted to 5.5 by adding solid $\mathrm{Na}_{2} \mathrm{CO}_{3}$.

LI of CTA. LI was assessed using a CTA paradigm adopted from ref. 35. It comprises a 1-trial conditioning paradigm in which a single pairing of a taste (sucrose solution) and gastric malaise ( $\mathrm{LiCl}$ injection) induces a lasting aversion to the taste of sucrose. During the experiment, the animals were maintained on a water deprivation regimen in order to motivate performance (35). The experiment consisted of 3 phases: preexposure, conditioning, and testing. In the preexposure phase, subjects in the PE condition were provided with $10 \%(\mathrm{w} / \mathrm{v}) \mathrm{D}$-sucrose solution, while subjects in the $\mathrm{nPE}$ condition had access to water. In the conditioning phase, 24 hours later, both $\mathrm{PE}$ and $\mathrm{nPE}$ subjects were provided with sucrose solution and received a $\mathrm{LiCl}$ injection 5 minutes afterward. In the test phase, another 24 hours later, CTA to the sucrose solution was measured in a 2-choice test, in which the animals could freely choose between sucrose solution and water.

T-maze test of delayed alternation. To motivate performance in the T-maze test, animals were maintained on a food restriction regime as described elsewhere (56). First, the animals received daily habituation to the T-maze, the food rewards, and the experimenter (15 minutes per day) for 5 days. Following this, alternation performance was assessed across 3 test sessions, with 2 days in between each test. Each session began with a forced trial in which the animal was released from the start arm and allowed access to only 1 out of 2 possible choice arms to obtain a food reward. This was followed by 10 choice trials in which the animals could choose between the 2 choice arms after being released from the start stem. They were rewarded for selecting the arm they had not visited in the previous trial, i.e., they had to learn to alternate between the 2 choice arms across trials, regardless of whether the previous trial led to a reward. An intertrial interval of $5 \mathrm{sec}-$ onds separated successive choice trials.

Statistics. Statistical analysis was performed with ANOVA followed by post-hoc Fisher's LSD pair-wise comparisons. $P<0.05$ was accepted as statistical significance. Values are expressed as mean \pm SEM.

Study approval. All animal procedures were conducted in a facility accredited by the Association for Assessment and Accreditation of Laboratory Animal Care in accordance with protocols approved by the Institutional Animal Care and Use Committee of the Legacy Research Institute and the principles outlined by the NIH.

\section{Acknowledgments}

This work has been supported by NIH grant R01MH083973.

Received for publication December 12, 2011, and accepted in revised form May 9, 2012.

Address correspondence to: Detlev Boison, Robert Stone Dow Neurobiology Laboratories, Legacy Research Institute, $1225 \mathrm{NE}$ 2nd Avenue, Portland, Oregon, USA. Phone: 503.413.1754; Fax: 503.413.5465; E-mail: dboison@downeurobiology.org.
2012;62(3):1527-1543.

5. Sebastião AM, Ribeiro JA. Adenosine receptors and the central nervous system. Handb Exp Pharmacol. 2009;(193):471-534.

6. Fredholm BB, Chen JF, Cunha RA, Svenningsson P, Vaugeois JM. Adenosine and brain function. Int Rev Neurobiol. 2005;63:191-270.

7. Wu LG, Saggau P. Presysnaptic inhibition of elicited neurotransmitter release [Review]. Trends in Neurosciences. 1997;20(5):204-212.

8. Thompson SM, Capogna M, Scanziani M. Pre- synaptic inhibition in the hippocampus. TINS. 1993;16(6):222-227.

9. Fuxe K, Ferré S, Genedani S, Franco R, Agnati LF. Adenosine receptor-dopamine receptor interactions in the basal ganglia and their relevance for brain function. Physiol Behav. 2007;92(1-2):210-217.

10. Franco R, et al. Receptor-receptor interactions involving adenosine A1 or dopamine D1 receptors and accessory proteins. J Neural Transm. 2007;114(1):93-104.

11. Brunstein $M G$, et al. Increased serum adenosine 
deaminase activity in schizophrenic receiving antipsychotic treatment. Neurosci Lett. 2007;414(1):61-64.

12. Dutra GP, Ottoni GL, Lara DR, Bogo MR. Lower frequency of the low activity adenosine deaminase allelic variant (ADA1*2) in schizophrenic patients. Rev Bras Psiquiatr. 2010;32(3):275-278.

13. Akhondzadeh S, Safarcherati A, Amini H. Beneficial antipsychotic effects of allopurinol as add-on therapy for schizophrenia: a double blind, randomized and placebo controlled trial. Prog Neuropsychopharmacol Biol Psychiatry. 2005;29(2):253-259.

14. Lara DR, Brunstein MG, Ghisolfi ES, Lobato MI, Belmonte-de-Abreu P, Souza DO. Allopurinol augmentation for poorly responsive schizophrenia. Int Clin Psychopharmacol. 2001;16(4):235-237.

15. Golembiowska K, Zylewska A. Agonists of A1 and $\mathrm{A} 2 \mathrm{~A}$ adenosine receptors attenuate methamphetamine-induced overflow of dopamine in rat striatum. Brain Res. 1998;806(2):202-209.

16. Ferre S. Adenosine-dopamine interactions in the ventral striatum. Implications for the treatment of schizophrenia. Psychopharmacology (Berl). 1997;133(2):107-120.

17. Ferre S, O'Connor WT, Snaprud P, Ungerstedt U, Fuxe K. Antagonistic interaction between adenosine $\mathrm{A} 2 \mathrm{~A}$ receptors and dopamine $\mathrm{D} 2$ receptors in the ventral striopallidal system. Implications for the treatment of schizophrenia. NeuroScience. 1994;63(3):765-773.

18. Shen H-Y, et al. A critical role of the adenosine A2A receptor in extra-striatal neurons in modulating psychomotor activity as revealed by opposite phenotypes of striatum- and forebrain-A2A receptor knockouts. J Neurosci. 2008;28(12):2970-2975.

19. Boison D. Modulators of nucleoside metabolism in the therapy of brain diseases. Curr Top Med Chem 2011;11(8):1068-1086

20. Boison D, Chen JF, Fredholm BB. Adenosine signalling and function in glial cells. Cell Death Differ. 2010;17(7):1071-1082.

21. Gouder N, Scheurer L, Fritschy J-M, Boison D. Overexpression of adenosine kinase in epileptic hippocampus contributes to epileptogenesis. J Neurosci. 2004;24(3):692-701.

22. Li T, et al. Adenosine kinase is a target for the prediction and prevention of epileptogenesis in mice. J Clin Inv. 2008;118(2):571-582.

23. Li T, Lan JQ, Fredholm BB, Simon RP, Boison $\mathrm{D}$. Adenosine dysfunction in astrogliosis: cause for seizure generation? Neuron Glia Biology. 2007;3(4):353-366

24. Shen HY, Lusardi TA, Williams-Karnesky RL, Lan JQ, Poulsen DJ, Boison D. Adenosine kinase determines the degree of brain injury after ischemic stroke in mice. J Cereb Blood Flow Metab. 2011;31(7):1648-1659.

25. Yee BK, Singer P, Chen JF, Feldon J, Boison D. Transgenic overexpression of adenosine kinase in brain leads to multiple learning impairments and altered sensitivity to psychomimetic drugs. Eur J Neurosci. 2007;26(11):3237-3252.

26. Huber A, Padrun V, Deglon N, Aebischer P, Mohler
H, Boison D. Grafts of adenosine-releasing cells suppress seizures in kindling epilepsy. Proc Natl Acad Sci U S A. 2001;98(13):7611-7616.

27. Jarvis MF, et al. ABT-702 (4-amino-5-(3bromophenyl)-7-(6-morpholinopyridin-3-yl) pyrido[2, 3-d]pyrimidine), a novel orally effective adenosine kinase inhibitor with analgesic and antiinflammatory properties: I. In vitro characterization and acute antinociceptive effects in the mouse. JPharmacol Exp Ther. 2000;295(3):1156-1164.

28. Braff DL, et al. Impact of prepulse characteristics on the detection of sensorimotor gating deficits in schizophrenia. Schizophr Res. 2001;49(1-2):171-178.

29. Braff DL, Geyer MA, Swerdlow NR. Human studies of prepulse inhibition of startle: normal subjects, patient groups, and pharmacological studies. Psychopharmacology (Berl). 2001;156(2-3):234-258.

30. Swerdlow NR, Weber M, Qu Y, Light GA, Braff DL. Realistic expectations of prepulse inhibition in translational models for schizophrenia research. Psychopharmacology (Berl). 2008;199(3):331-388.

31. Singer P, Feldon J, Yee BK. Are DBA $/ 2$ mice associated with schizophrenia-like endophenotypes? A behavioural contrast with C57BL/6 mice. Psychopharmacology (Berl). 2009;206(4):677-698.

32. Masino SA, et al. A ketogenic diet suppresses seizures in mice through adenosine A1 receptors. J Clin Inv. 2011;121(7):2679-2683.

33. Lubow RE, Kaplan O, Abramovich P, Rudnick A, Laor N. Visual search in schizophrenia: latent inhibition and novel pop-out effects. Schizophr Res. 2000;45(1-2):145-156

34. Weiner I. The "two-headed" latent inhibition model of schizophrenia: modeling positive and negative symptoms and their treatment. Psychopharmacology (Berl). 2003;169(3-4):257-297.

35. Meyer U, Chang DL, Feldon J, Yee BK. Expression of the CS- and US-pre-exposure effects in the conditioned taste aversion paradigm and their abolition following systemic amphetamine treatment in C57BL6/J mice. Neuropsychopharmacology. 2004;29(12):2140-2148.

36. Boison D. Adenosine-based cell therapy approaches for pharmacoresistant epilepsies. Neurodegener Dis. 2007;4(1):28-33.

37. Lara DR, Dall'Igna OP, Ghisolfi ES, Brunstein MG. Involvement of adenosine in the neurobiology of schizophrenia and its therapeutic implications. Prog Neuropsychopharmacol Biol Psychiatry. 2006;30(4):617-629.

38. Wang JH, Short J, Ledent C, Lawrence AJ, van den Buuse M. Reduced startle habituation and prepulse inhibition in mice lacking the adenosine A2A receptor. Behav Brain Res. 2003;143(2):201-207.

39. Fedele DE, et al. Astrogliosis in epilepsy leads to overexpression of adenosine kinase resulting in seizure aggravation. Brain. 2005;128(pt 10):2383-2395.

40. Pignataro G, Simon RP, Boison D. Transgenic overexpression of adenosine kinase aggravates cell death in ischemia. J Cereb Blood Flow Metab. 2007;27(1):1-5.

41. Arguello PA, Gogos JA. Modeling madness in mice: one piece at a time. Neuron. 2006;52(1):179-196.

42. Durieux PF, Schiffmann SN, de Kerchove d'Exaerde A. Differential regulation of motor control and response to dopaminergic drugs by D1R and D2R neurons in distinct dorsal striatum subregions. EMBO J. 2012;31(3):640-653.

43. Fuxe K, Marcellino D, Leo G, Agnati LF. Molecular integration via allosteric interactions in receptor heteromers. A working hypothesis. Curr Opin Pharmacol. 2010;10(1):14-22.

44. Fredholm BB, Svenningsson P. Adenosine-dopamine interactions: development of a concept and some comments on therapeutic possibilities. Neurology. 2003;61(11 suppl 6):S5-S9.

45. Chen JF, Yu L, Shen HY, He JC, Wang X, Zheng R. What knock-out animals tell us about the effects of caffeine. J Alzheimers Dis. 2010;20 suppl 1:S17-S24.

46. Lubow RE. Construct validity of the animal latent inhibition model of selective attention deficits in schizophrenia. Schizophr Bull. 2005;31(1):139-153.

47. Chen JF, et al. Selective attenuation of psychostimulant-induced behavioral responses in mice lacking A(2A) adenosine receptors. NeuroScience. 2000;97(1):195-204.

48. Wei CJ, Singer P, Boison D, Yee BK, Chen JF. Selective inactivation of adenosine $\mathrm{A} 2 \mathrm{~A}$ receptors in striatal neurons enhances working memory and reversal learning. Learn Mem. 2011;18(7):459-474.

49. Freeman JM, Kossoff EH, Hartman AL. The ketogenic diet: one decade later. Pediatrics. 2007;119(3):535-543.

50. Kraft BD, Westman EC. Schizophrenia, gluten, and low-carbohydrate, ketogenic diets: a case report and review of the literature. Nutr Metab (Lond). 2009;6:10.

51. Akhondzadeh S, Shasavand E, Jamilian H, Shabestari O, Kamalipour A. Dipyridamole in the treatment of schizophrenia: adenosine-dopamine receptor interactions. J Clin Pharm Ther. 2000;25(2):131-137.

52. Boison D. Adenosine augmentation therapies (AATs) for epilepsy: prospect of cell and gene therapies. Epilepsy Res. 2009;85(2-3):131-141.

53. Boison D, Stewart KA. Therapeutic epilepsy research: from pharmacological rationale to focal adenosine augmentation. Biochem Pharmacol. 2009;78(12):1428-1437.

54. Yee BK, Chang T, Pietropaolo S, Feldon J. The expression of prepulse inhibition of the acoustic startle reflex as a function of three pulse stimulus intensities, three prepulse stimulus intensities, and three levels of startle responsiveness in C57BL6/J mice. Behav Brain Res. 2005;163(2):265-276.

55. Csomor PA, Stadler RR, Feldon J, Yee BK, Geyer MA, Vollenweider FX. Haloperidol differentially modulates prepulse inhibition and p50 suppression in healthy humans stratified for low and high gating levels. Neuropsychopharmacology. 2008;33(3):497-512.

56. Zhou SJ, et al. Preferential enhancement of working memory in mice lacking adenosine $\mathrm{A}(2 \mathrm{~A})$ receptors. Brain Res. 2009;1303:74-83. 